\title{
A limit of the subjective age bias: Feeling younger to a certain degree, but no more, is beneficial for life satisfaction
}

\author{
Maria Blöchl \\ University of Münster \& Max Planck Institute \\ for Human Brain and Cognitive Sciences
}

\author{
Steffen Nestler \\ University of Münster
}

\author{
David Weiss \\ University of Leipzig
}

\begin{abstract}
The majority of adults feels considerably younger than their chronological age. Numerous studies suggest that maintaining a younger subjective age is linked to greater life satisfaction. However, whether there is a limit beyond which feeling younger becomes detrimental is not well understood. Here, we use response surface analysis to examine the relationships between subjective age, chronological age, and life satisfaction in in a large sample spanning adulthood $(N=7,356 ; 36-89$ years). We find that there is a limit to feeling younger: People who feel younger by a certain amount, but not more, have the highest levels of life satisfaction. In addition, our findings suggest that the discrepancy between subjective and chronological age at which life satisfaction is highest increases across the adult age span. Taken together, these findings reveal that beyond a certain point, feeling younger than one's chronological age may be psychologically harmful.
\end{abstract}

Keywords: subjective age, aging, life satisfaction, well-being, response surface analysis

There is ample evidence that as people grow older, they do not necessarily feel older (Montepare \& Lachman, 1989). Beyond the age of 40 years, about $70 \%$ of adults feel considerably younger than their chronological age (Chopik \& Giasson, 2017; Rubin \& Berntsen, 2006). This pervasive tendency to report a younger subjective age has been referred to as the subjective age

Maria Blöchl, Department of Psychology, University of Münster \& Department of neurology, Max Planck Institute for $\mathrm{Hu}-$ man Cognitive and Brain Sciences; Steffen Nestler, Department of Psychology, University of Münster; David Weiss, Department of Psychology, University of Leipzig.

We preregistered our hypotheses and analyses plan on the Open Science Framework (OSF; https://osf.io/fsjg4/). In addition, we have provided our analyses scripts on OSF (https://osf.io/kg84e/). Preliminary findings reported in this manuscript have been presented at the PaEpsy Meeting, September 2019 in Leipzig, Germany and a preprint was posted on PsyArxiv at https://psyarxiv.com/pfxqh/ (Blöchl et al., 2020).

Correspondence concerning this paper should be addressed to Maria Blöchl, Department of Psychology, University of Münster, Fliednerstr. 21, 48149 Münster, Germany; Tel: +492518334127; Email: mbloechl@uni-muenster.de. bias $^{1}$ (Weiss \& Weiss, 2019). Previous research consistently linked a younger subjective age bias to a myriad of positive outcomes. Those who feel younger, compared to those who feel older, experience higher psychological well-being, less stress, fewer depressive symptoms, better physical health, better cognitive performance, and even lower mortality (Armenta et al., 2018; Barak \& Stern, 1986; Choi \& DiNitto, 2014; Stephan et al., 2018a; Weiss \& Lang, 2012; Westerhof \& Barrett, 2005; Zee \& Weiss, 2019; for an overview see Alonso Debreczeni \& Bailey, 2020; Kotter-Grühn et al., 2015, and Westerhof et al., 2014). Although these findings have spurred the notion that a young subjective age can be conceptualized as a biopsychosocial marker of healthy ageing (Stephan et al., 2015; Westerhof et al., 2014), it is critical to question whether feeling younger is indeed always better. Is it, for example, beneficial to feel 30 years old when one is 85 years old? Or is there a limit beyond which feeling younger becomes less good, or even detrimental?

\footnotetext{
${ }^{1}$ We use the term 'subjective age bias' because the age people feel represents on average a deviation from their chronological age.
} 
Given that older ages are often associated with undesirable expectations such as loss and decline (Heckhausen et al., 1989), it is argued that the subjective age bias has an adaptive function: it allows older adults to maintain a positive self-perception by distancing themselves from the negative views of ageing. Thus, feeling younger may serve a self-protective function and promotes well-being (Teuscher, 2009; Ward, 1977; Weiss \& Kornadt, 2018). Accordingly, experiments have shown that older adults tend to distance themselves from their age group and reporting a younger subjective age when they are confronted with negative age-related information, which can help protect their self-concept (Armenta et al., 2018; Weiss \& Freund, 2012; Weiss \& Lang, 2012).

However, increasing evidence is challenging the notion that a young subjective age is always beneficial. For example, older adults who try hard to appear younger (e.g. using invasive concealment procedures or adopting youth-centric resources) are more negatively evaluated (Chasteen et al., 2011; North \& Fiske, 2013; Schoemann \& Branscombe, 2011). In addition, there is evidence that idealizing and romanticizing youthfulness can impair older adults' recovery from physical illness (Levy et al., 2019). Further, studies have shown that exaggerated expectations concerning one's future well-being can have detrimental consequences in later adulthood (Lachman et al., 2008; Lang et al., 2013). At a certain point, distancing oneself from one's own age might therefore become psychologically harmful (Weiss \& Kornadt, 2018).

A plausible alternative hypothesis is that there is a limit to feeling younger: Individuals are happiest when their subjective age is younger than their actual age to a certain degree, but not more (Baumeister, 1989; Gana et al., 2004). Research shows that even if older adults often feel younger than their age peers, they feel more similar to middle-aged than younger adults (Weiss \& Freund, 2012). A moderate subjective age bias may be optimal because it may provide benefits, such as fostering a realistic motivation and confidence to maintain work performance and leisure activities (Armenta et al., 2018; Cleaver \& Muller, 2002). In contrast, larger subjective age biases may be psychologically and socially stressful to maintain. Moreover, given that older age is associated with increasingly negative expectations (Heckhausen et al., 1989; Weiss \& Lang, 2012), it is also plausible to assume that a limit to feeling younger is not fixed but increases throughout adulthood.

Evidence for a limit beyond which an individual's subjective age bias is detrimental is still lacking. To date, the vast majority of studies focused on perspectives that assume that feeling young(er) is always better. More specifically, previous work has mainly relied on regression analyses that only account for linear effects of (i) subjective age (i.e. people with the youngest subjective age enjoy the most positive outcomes) or (ii) the difference between subjective and chronological age (i.e. people with the highest discrepancy between subjective and chronological age enjoy the most positive outcomes). However, these approaches cannot capture potential non-linear effects. Thus, they prohibit to draw any conclusions about a limit beyond which maintaining a younger subjective age becomes detrimental.

Only two previous studies have examined the potential limitations of feeling young for psychological outcomes (Gana et al., 2004; Palgi et al., 2018). Both studies reported no evidence that low self-esteem and depressive symptoms are more common in people who feel extremely younger than their chronological age compared to those who feel moderately younger (Gana et al., 2004; Palgi et al., 2018). However, these studies relied on categorizing (proportional) difference scores of subjective age and chronological age, which is problematic for two reasons. First, difference scores conceal information on the levels of both variables when they are collapsed into one score (Edwards, 2001, 2002). Hence, difference scores only allow testing of a limited number of hypotheses on subjective age. For example, they cannot be used to reveal potentially important agedifferential effects of feeling younger, which may be theoretically meaningful from a lifespan perspective (Barrett \& Montepare, 2015; Humberg et al., 2019). Second, categorizing scores is prone to bias because it leads to further loss of information and statistical power to detect non-linear relations (Altman \& Royston, 2006; DeCoster et al., 2009; Royston et al., 2006). These methodological shortcomings preclude any strong conclusions about the precise relationship between subjective age, chronological age, and life satisfaction.

\section{The Current Study}

In the current study, we sought to examine whether there is a limit up to which feeling younger is linked to higher life satisfaction and how this limit might change across the adult lifespan. We analyzed data from a large, age-heterogenous sample of adults $(N=7,356$; $36-89$ years), who responded to questions about their subjective age and life satisfaction. To overcome the 
methodological limitations of previous research, we analyzed the data using response surface analysis (RSA) in combination with a model selection approach. RSA is a two-stepped procedure comprising (1) the fit of polynomial regression model, and then (2) using coefficients from the regression model to plot a response surface illustrating the relationships among all variables (Edwards, 2002; Humberg, Dufner, et al., 2019; Humberg, Nestler, et al., 2019; Schönbrodt, 2016). RSA preserves subjective and chronological age as separate predictors and does not collapse them, such as difference scores. As such, RSA allows us to go beyond previous research because it allows to formalize commonly tested relations (e.g. a linear regression using difference scores) as well as more complex relations between subjective age, chronological age, and life satisfaction. Using a model selection approach, these different formalizations can then be tested against each other using the same data.

We first consider three theoretical perspectives that make different predictions about whether there is a limit up to which feeling younger is beneficial to life satisfaction and whether this limit changes across the adult lifespan: the young(er)-is-better perspective, the optimal margin perspective, and the increasing optimal margin perspective. All three perspectives and their variants are depicted in Figure 1 (see Methods section and Table 1 for more details). Critically, these perspectives make distinct predictions for people who maintain an extremely younger subjective age. The young(er)-isbetter perspective is consistent with most previous research on subjective age and life satisfaction and entails two variants. The first variant makes the unique prediction that adults who feel young, no matter their chronological age, have the highest levels of life satisfaction (Figure 1a). The second variant makes the unique prediction that those who feel younger (i.e. have the highest discrepancy between subjective and chronological age as typically tested using a difference score approach) have the highest level of life satisfaction (Figure 1b). The optimal margin perspective, in contrast, predicts that there is a limit to feeling younger: feeling younger to a certain amount - but not too much - is linked to maximal life satisfaction (Figure 1c and 1d). Finally, the increasing optimal margin perspective predicts that there is a limit beyond which feeling younger ceases to be beneficial but, importantly, considers a lifespan perspective to subjective ageing (Barrett \& Montepare, 2015). Additionally, it suggests that the limit of feeling younger increases across the adult lifespan (Figure 1e and 1f).
All three perspectives can be formalized within the RSA framework (see Table 1). We fit all competing models to data from a large sample spanning adulthood $(N=7,356 ; 36-89$ years) and apply a model selection approach to compare the relative evidence for these competing perspectives within the same data set (Burnham \& Anderson, 2004; Humberg, Dufner, et al., 2019; Schönbrodt, 2016; Symonds \& Moussalli, 2011). Hence, we are able to discern which perspective is best supported by the data. In other words, we were directly able to quantitatively compare how well the data supported the suggestion that there is a downside to feeling younger compared to the suggestion that there is a linear relationship between feeling young(er) and life satisfaction found in most previous studies.

\section{Method}

Our main hypotheses and our modelling strategy were preregistered on the Open Science Framework (OSF; https://osf.io/fsjg4/) before any analyses were performed. Analyses scripts have also been made available on the accompanying OSF project website (https://osf.io/kg84e/).

\section{Participants}

This study used data from the English Longitudinal Study of Ageing (ELSA). ELSA is an ongoing, population-based study to investigate the health and ageing of men and women England. A detailed description of ELSA's sample and sampling procedure can be found elsewhere (Steptoe et al., 2013). In brief, the study started in 2002 (Wave 1) by recruiting a nationally representative sample of people aged 50 or older from the annual cross-sectional household survey (Health Survey for England; HRS); all cohabitating partners, some of which were younger, were also eligible to take part in ELSA. All participants are interviewed every two years and the most recent wave of data collection (Wave 8) was completed in 2017. For the purpose of the current study, we analyzed data from Wave 2 (2004 - 2005), since this was the first assessment that included measures of subjective age and life satisfaction. Of the 9,432 participants in Wave 2, we restricted our analyses to individuals who provided data on chronological age, subjective age, and life satisfaction $(N=$ $7,419)$. In this step, participants who were 90 years or older at the time of the interview had to be excluded since their chronological age was collapsed to 99 years 
Young(er)-is-better perspective

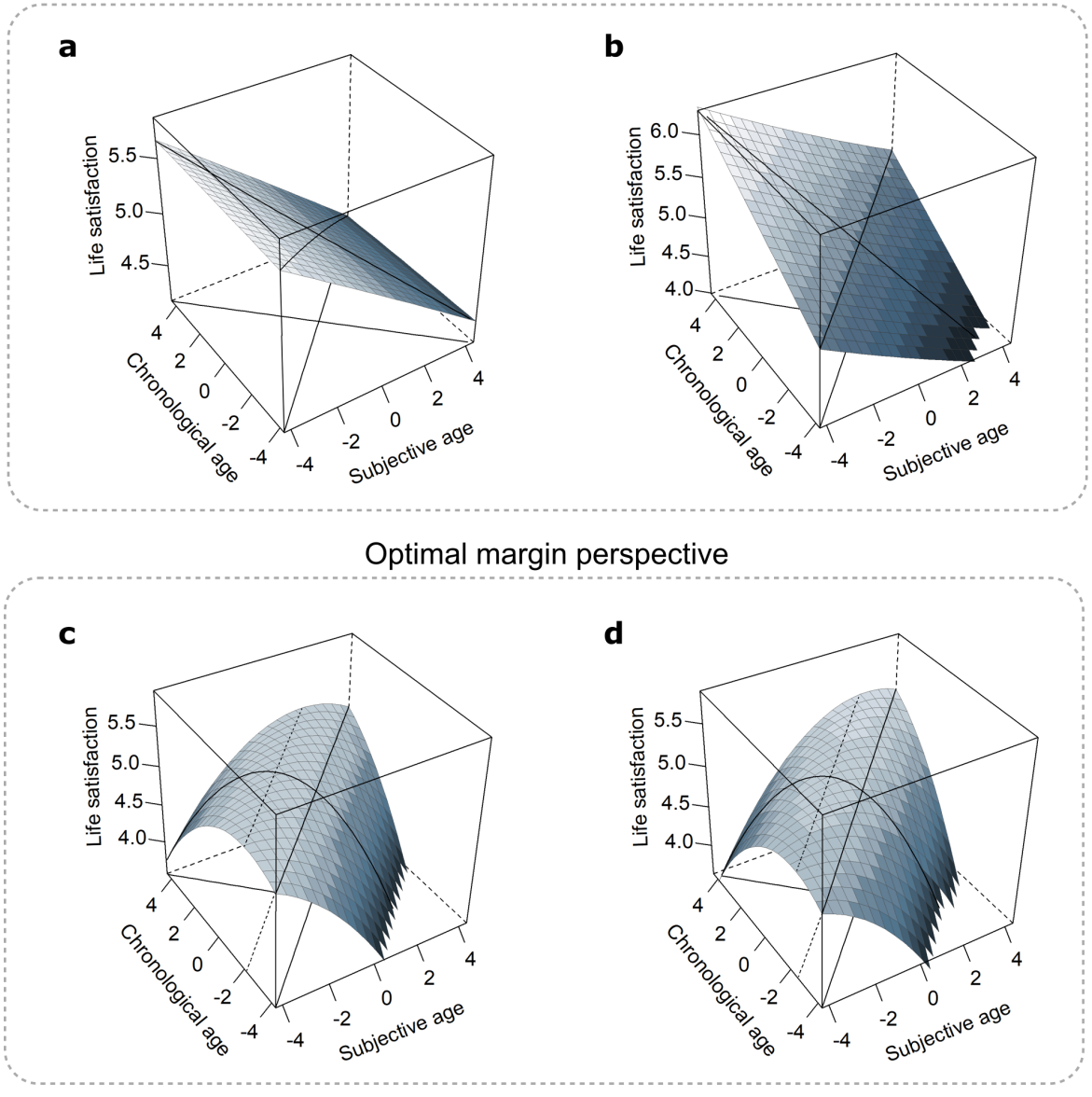

Increasing optimal margin perspective

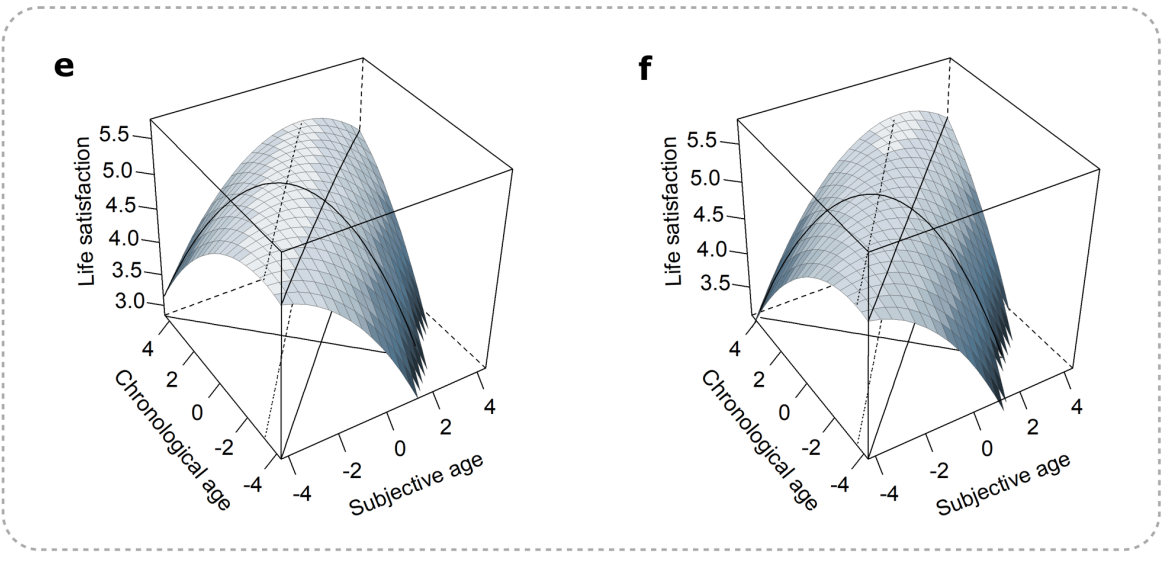

Figure 1. Illustrations showing the response surface graphs of the three main perspectives. The response surface graphs plot the associations between associations between subjective age ( $\mathrm{x}$ axis; bottom right), chronological age (y-axis; bottom left), and life satisfaction (z-axis; vertical). The surface is color-coded so that lighter shades indicate higher levels of life satisfaction and darker shades the lower levels. The black solid line, which runs from the corner in the front to the corner in the back depicts the line of congruence (LOC), that is the values of life satisfaction on the response surface that are predicted if people feel exactly their age (i.e. have no subjective age bias). The dotted line in the four lower graphs, depicts the ridge. The ridge indicates the respective combination of chronological and subjective age that are linked to the highest levels of life satisfaction. In these models, the ridge is shifted to the left of the LOC, suggesting that 
there is a limit associated with feeling younger: that feeling younger, but only a certain extent, is linked to maximal levels of life satisfaction. Moreover, the ridge can be tilted (i.e. not parallel to Figure 1 (continued). the $\mathrm{x}-\mathrm{y}$ plane; as in $\mathrm{d}$ and $\mathrm{f}$ ), suggesting additional main effects of chronological and subjective age. Finally, the ridge can be rotated (i.e. not parallel to the LOC; as in e and f), which suggests an increasing discrepancy of subjective and chronological age across the adult lifespan that is related to life satisfaction: a. young subjective age-only (hypothesis 1.a); b. young subjective and older chronological age (hypothesis 1.b); c. optimal margin-only (hypothesis 2.a); d. optimal margin with main effects (hypothesis 2.b) e. increasing optimal margin-only (hypothesis 3.a); f. increasing optimal margin with main effects (hypothesis 3.b)

by the ELSA team to prevent identification. After submitting the preregistration, we also decided to prevent bias that might arise from misunderstanding the instructions by excluding participants with a subjective age above or below $3 S D$ of the sample's mean (e.g. subjective age of 0 or 120 years; $N=53$; see e.g. Stephan et al., 2015); the same exclusion criterion was applied to chronological age $(N=10)$, resulting in a final sample of 7,356 participants (the results of our analyses applying the preregistered exclusion criteria are reported in Supplementary Results 1, Table S1; they were similar to our main results reported here).

\section{Measures}

Chronological age. Information on participants' chronological age (in years) was provided by the ELSA team and computed from the difference between participants' date of birth and the date of their individual interview $(M=64.40$ years, $S D=9.57)$.

Subjective age. Subjective age was assessed by asking participants: 'How old do you feel that you are?' Participants then reported the age they felt in years $(M$ $=55.35$ years, $S D=12.9$ ). For descriptive analyses, we also calculated the discrepancy between subjective age and chronological age, with negative values indicating that people feel younger than their chronological age and positive values indicating that people feel older than their chronological age.

Satisfaction with life. Subjective well-being was measured using the Satisfaction with Life Scale (Diener et al., 1985), which consists of five items about overall life satisfaction (e.g. "In most ways my life is close to ideal", "I am satisfied with my life."). Answers were given on a seven-point Likert scale ranging from 1 ("strongly agree") to 7 ("strongly disagree"). All items were coded so that higher values represent higher levels of life satisfaction and averaged to a mean score for each participant (Cronbach's alpha $=.90)$.

\section{Modelling framework}

We used RSA to analyze the data. This approach overcomes the limitations of previous research (e.g. the use of difference scores and categorization of predictors; see Edwards, 2002; Humberg et al., 2019; Schönbrodt, 2016). It enables us to accurately model how different combinations of subjective age and chronological age relate to life satisfaction. The full polynomial regression model represents life satisfaction as a function of subjective age and chronological age, and incorporates their quadratic and interaction terms:

$$
\begin{aligned}
\text { life satisfaction } & =b_{0}+b_{1} \times \text { subjective age } \\
& +b_{2} \times \text { chronological age } \\
& +b_{3} \times \text { subjective age }{ }^{2} \\
& +b_{4} \times \text { subjective age } \\
& \times \text { chronological age } \\
& +b_{5} \times \text { chronological age }{ }^{2}
\end{aligned}
$$

By placing specific constraints on the regression coefficients of the full polynomial model, we translated our competing perspectives on the limitations of feeling younger across adulthood into distinct statistical models (Table 1$).^{2}$ Figure 1 provides a graphical depiction of the response surfaces all hypotheses. The shape of the response surface in the three-dimensional coordinate system reflects the predicted relationships among subjective age, chronological age, and life satisfaction of each perspective. For the first perspective, the predicted response surfaces are planes, whereas for the second and third perspective, they take the shape of a saddle (see Figure 1). To aid interpretation of the response surfaces, we also plotted three properties: the

\footnotetext{
${ }^{2}$ We note that after submitting the preregistration, we decided to change the naming of some models for understandability. We also corrected an error in the specification of model 3.a and included model specifications with a rising ridge (main effects) for the optimal margin models (model 2.b and model 3.b). In light of the extant literature (Schönbrodt, 2015), their omission was a clear oversight in our preregistration.
} 
line of congruence (LOC), the line of incongruence (LOIC), and the ridge. The LOC (solid black line) runs from the front corner to the back corner of the cube. It indicates the position of congruent chronological age and subjective age on the response surface. The LOIC (solid black line) is perpendicular to the LOC and indicates the position of combinations in which chronological age and subjective age are equal in magnitude, but opposite in sign. The ridge (dotted line) is a crucial feature of the response surface if it is shaped like a saddle. It indicates which combination of chronological and subjective age are linked to the highest levels of life satisfaction. Details on the different models, their constrains, and their response surface are described in the following.

Young(er)-is-better perspective. This perspective posits that a young subjective age is associated with higher life satisfaction, beyond the effects of chronological age. We consider two variants of this perspective.

Young subjective age-only (hypothesis 1.a): The young subjective age-only hypothesis is consistent with studies that show a linear relationship between subjective age and life satisfaction using correlation or regression analyses based on subjective age. It posits that people with a young subjective age - regardless of their chronological age - have the highest life satisfaction. Thus, subjective age is negatively and linearly associated with life satisfaction, while chronological age has no effect beyond subjective age. There are no quadratic or interaction effects. This hypothesis is depicted in Figure 1a.

Young subjective and older chronological age (hypothesis 1.b): The young subjective and older chronological age hypothesis is related to the idea that the difference between subjective and chronological age (e.g. using difference scores) is linearly associated with life satisfaction. ${ }^{3}$ It posits that people most satisfied with their lives are those with a young subjective age and a high chronological age (i.e. the highest discrepancy). Thus, subjective age is negatively associated with life satisfaction, while chronological age is positively associated with life satisfaction, independently of one another. There are no quadratic or interaction effects. This hypothesis is depicted in Figure 1b.

Optimal margin perspective. The optimal margin hypothesis posits that there are downsides to feeling "too" young. This perspective is based on the classical optimal margin of illusion theory, which claims that people whose (self-)perceptions only deviate slightly from reality enjoy the highest well-being (Baumeister,
1989). Applied to the realm of subjective age, the optimal margin perspective suggests that life satisfaction is maximized for a specific discrepancy between subjective age and chronological age (Gana et al., 2004; Palgi et al., 2018). One important assumption of the optimal margin perspective is that there is a fixed discrepancy for which life satisfaction is maximized across adulthood. ${ }^{4}$ This perspective is represented by two variants.

Optimal margin hypothesis-only (hypothesis 2.a). The optimal margin-only hypothesis assumes that a certain discrepancy between subjective age and chronological age, but no more, is linked to life satisfaction. Subjective and chronological age are not related to life satisfaction beyond their discrepancy effect, which is fixed across adulthood. The discrepancy effect is reflected in the parameter $C \neq 0$, which indicates a shift of the ridge from the line of congruence (LOC). Given that the discrepancy $(C)$ is assumed to be fixed across adulthood, the ridge of the response surface is not rotated but parallel to the LOC $(S=1)$. Moreover, there are no main effects and the ridge is flat and therefore

\footnotetext{
${ }^{3}$ The hypothesis is, in fact, a less restrictive version of the standard difference score approach, which is often used in the literature and in which chronological age is distracted from subjective age (or vice versa) to be used as a regressor. Model 1.b included here constrains $b_{1}$ and $b_{2}$ to being of opposite value, which would also be implied by the standard difference score model. However, in contrast to a classical difference score model, $b_{1}$ and $b_{2}$ in our model are not constrained to being of the same value. In comparison, the standard difference score model, as often applied in the subjective age literature, would be defined as follows: life satisfaction $=b_{0}+b_{1} \times$ subjective age $+b_{2} \times$ chronological age $+b_{3} \times$ subjective age ${ }^{2}+b_{4} \times$ subjective age $\times$ chronological age $+b_{5} \times$ chronological age ${ }^{2}$, with $b_{1}=-b_{2}$ and $b_{3}=b_{4}=b_{5}=0$. Given that the difference score model (hypothesis 1.b) included here (which is, as previously stated, a less restrictive version of the standard difference score model; see Table 1) is not well supported by the data and fits less well the optimal margin models, it follows that the more restrictive standard difference score model must as well. In fact, fitting this standard difference score model revealed a particularly poor fit $\left(\mathrm{AIC}=23712.87 ; \mathrm{CFI}=0.539 ; R^{2}=0.022\right)$, comparable to model 1.b, and much worse than the best fitting optimal margin models (see Table 1).

${ }^{4}$ Please note that this perspective is different from a perspective that proposes and models a quadratic effect of the discrepancy between subjective and chronological age. A model that represents a quadratic relationship between feeling younger and life satisfaction has also been included in the model set; it is represented by supplementary model 6 . This model would actually predict that people whose subjective age is congruent with their actual age have the highest life satisfaction. As expected, this model performs less well than the optimal margin models, which further supports the notion that the RSA approach captures these relationships better than an approach that relies solely on modelling a squared difference score.
} 
Table 1

Description of all Hypotheses, Their Respective Constraints Imposed on the Full Polynomial Regression Model, and Surface Parameters

\begin{tabular}{|c|c|c|c|}
\hline Hypothesis & Interpretation & Model constraints & $\begin{array}{c}\text { Specific surface } \\
\text { parameters }\end{array}$ \\
\hline \multicolumn{4}{|c|}{ Young(er)-is-better perspective } \\
\hline $\begin{array}{l}\text { 1.a) Young } \\
\text { subjective age-only } \\
\text { hypothesis }\end{array}$ & $\begin{array}{l}\text { People who feel young have } \\
\text { higher life satisfaction, } \\
\text { regardless of their } \\
\text { chronological age. }\end{array}$ & $\begin{array}{l}b_{1}<0 ; b_{2}=0 ; b_{3}= \\
0 ; b_{4}=0 ; b_{5}=0\end{array}$ & \\
\hline $\begin{array}{l}\text { 1.b) Young } \\
\text { subjective and older } \\
\text { chronological age } \\
\text { hypothesis }\end{array}$ & $\begin{array}{l}\text { People who feel younger } \\
\text { than their chronological age } \\
\text { (higher discrepancy) have } \\
\text { higher life satisfaction. }\end{array}$ & $\begin{array}{l}b_{1}<0 ; b_{2}>0 ; b_{3}= \\
0 ; b_{4}=0 ; b_{5}=0\end{array}$ & \\
\hline \multicolumn{4}{|c|}{ Optimal margin perspective } \\
\hline $\begin{array}{l}\text { 2.a) Optimal margin- } \\
\text { only hypothesis }\end{array}$ & $\begin{array}{l}\text { People with a specific } \\
\text { discrepancy of subjective and } \\
\text { chronological age have the } \\
\text { highest life satisfaction. }\end{array}$ & $\begin{array}{l}b_{1}=-b_{2} ; b_{3}<0 ; b_{3}= \\
b_{5} ; b_{4}=-2 b_{5}\end{array}$ & $C:=b_{1} /\left(2 \times b_{3}\right)$ \\
\hline $\begin{array}{l}\text { 2.b) Optimal margin } \\
\text { with main effects } \\
\text { hypothesis }\end{array}$ & $\begin{array}{l}\text { People with a specific } \\
\text { discrepancy of subjective and } \\
\text { chronological age are most } \\
\text { satisfied with their lives, and } \\
\text { subjective and chronological } \\
\text { age have additional main } \\
\text { effects. }\end{array}$ & $b_{3}=b_{5} ; b_{4}=-2 b_{5}$ & $\begin{array}{l}C:=\left(b_{2}-b_{1}\right) /(4 \times \\
\left.b_{3}\right) \\
b_{\mathrm{M}}:=b_{1}+b_{2}\end{array}$ \\
\hline \multicolumn{4}{|c|}{ Increasing optimal margin perspective } \\
\hline $\begin{array}{l}\text { 3.a) Increasing } \\
\text { optimal margin-only } \\
\text { hypothesis }\end{array}$ & $\begin{array}{l}\text { People with a specific } \\
\text { discrepancy of subjective and } \\
\text { chronological age are most } \\
\text { satisfied with their lives, and } \\
\text { this optimal margin increases } \\
\text { across adulthood. }\end{array}$ & $\begin{array}{l}b_{1}=\left(b_{2} \times b_{4}\right) / 2 \times b_{5} \\
b_{4}{ }^{2}=4 \times b_{3} \times b_{5}\end{array}$ & $\begin{aligned} C & :=\frac{1}{2}\left(b_{2} / b_{5}\right) \\
S & :=-b_{4} /\left(2 \times b_{5}\right) .\end{aligned}$ \\
\hline $\begin{array}{l}\text { 3.b) Increasing } \\
\text { optimal margin with } \\
\text { main effects } \\
\text { hypothesis }\end{array}$ & $\begin{array}{l}\text { People with a specific } \\
\text { discrepancy of subjective and } \\
\text { chronological age are most } \\
\text { satisfied with their lives, } \\
\text { while this optimal margin } \\
\text { increases across adulthood } \\
\text { and subjective and } \\
\text { chronological age have } \\
\text { additional main effects. }\end{array}$ & $b_{4}^{2}=4 \times b_{3} \times b_{5}$ & $\begin{array}{l}C:=-\left(2 \times b_{1} \times\right. \\
\left.b_{5}+b_{2} \times b_{4}\right) /(4 \times \\
\left.b_{4} \times b_{5}\right) \\
S:=-b_{4} /\left(2 \times b_{5}\right) \\
b_{\mathrm{M}}:=b_{1} / S+b_{2}\end{array}$ \\
\hline
\end{tabular}

Note. The table shows information on response surface parameters that are crucial for differentiating and interpreting the optimal margin models (for an overview of surface parameters see e.g. Humberg, Nestler \& Back, 2019). $C=$ parameter reflecting the shift of the ridge; $S=$ parameter reflecting the rotation of the ridge; $b M=$ parameter reflecting the main effects. 
parallel to the $\mathrm{x}-\mathrm{y}$ plane $\left(b_{\mathrm{M}}=0\right)$. This hypothesis is depicted in Figure 1c.

Optimal margin hypothesis with main effects (hypothesis 2.b). As a variant of the optimal margin perspective, this hypothesis suggests that in addition to a fixed optimal margin across adulthood $(C \neq 0)$, there is a main effect of both predictors. In other words, a certain combination of subjective age and chronological age is independently linked to life satisfaction: those who are older and therefore feel relatively old are even more satisfied with their life than those who are younger and therefore feel relatively young. Consequently, the ridge of the response surface is not flat, but tilted $\left(b_{\mathrm{M}} \neq 0\right)$. Given that the optimal margin is still assumed to be fixed across adulthood, the ridge is not rotated $(S=1)$. This hypothesis is depicted in Figure $1 d$.

Increasing optimal margin perspective. The increasing optimal margin hypothesis is an extension of the optimal margin hypothesis. It posits that life satisfaction is maximized for a specific discrepancy between subjective age and chronological age and additionally takes a lifespan perspective. This perspective assumes that the discrepancy is not fixed but increases across adulthood. In other words, feeling younger is beneficial up to a certain point and this limit is larger for older adults. This perspective is represented by two variants (see Table 1 and Figure S1).

Increasing optimal margin hypothesis-only (hypothesis 3.a). The increasing optimal margin-only hypothesis suggests that there is a certain discrepancy between subjective age and chronological age at which life satisfaction is highest $(C \neq 0)$ and that this optimal margin increases for increasing values of chronological age $(S \neq 1)$. It also assumes that there is no main effect, so that the ridge is flat and therefore parallel to the $x-y$ plane $\left(b_{\mathrm{M}}=0\right)$. This hypothesis is depicted in Figure 1e.

Increasing optimal margin hypothesis with main effects (hypothesis 3.b). This variant of the increasing optimal margin perspective also suggests that there is a certain discrepancy at which life satisfaction is highest $(C \neq 0)$ and that this optimal margin increases for increasing values of chronological age $(S \neq 1)$. Additionally, it assumes that there is a main effect, so that the ridge is tilted (i.e. not parallel to the $\mathrm{x}-\mathrm{y}$ plane; $b_{\mathrm{M}} \neq 0$ ). This hypothesis is depicted in Figure 1f.

Complete model set. When conducting model comparisons, it is generally recommended to include all theoretically defensible models in the set of models (Burnham et al., 2011). Although we deemed the six models described above the most plausible, we ensured that we did not miss a potentially better, alternative model by specifying six other potentially relevant models (models S1 to S6). The supplementary models were also included in the model set and are shown in Figure S1. Thus, our complete model set consists of 12 hypothesized models, the full polynomial model, and the null model. It is important to note that all models are nested in the full polynomial model and that some models are nested within each other. An overview of the nesting structure in the complete model set is provided in Figure S2.

\section{Model fit and model selection}

All models were estimated using the structural equation modelling (SEM) software lavaan in R (version 3.5.1), using ML estimation with robust standard errors. Before estimating the models, we standardized both predictor variables, that is subjective and chronological age, using their grand mean and their pooled standard deviation. In addition to our pre-registered analyses, we also checked for multicollinearity. Variance inflation factors (VIFs) were computed for the predictors of the full polynomial model and indicated that multicollinearity was not an issue in the subsequent analyses (all VIFs $\leq 6$ ).

The main analyses were conducted in two steps. In the first step, we estimated the full model (all parameters of the polynomial regression were estimated freely) to check for a meaningful association between the predictors and life satisfaction because model comparisons are only plausible if the $\mathrm{R}^{2}$ of the full model is significant. In the second step, we estimated all models in the model set. The fit of the models was compared with Akaike's information criterion (AIC). In general, the AIC is an estimator for the relative quality of a statistical model for a given data set. While the AIC rewards goodness of fit, it also includes a penalty term for the number of estimated parameters in order to avoid overfitting. Given a set of competing models, their AICs provide an index of the quality of each model, relative to each of the other models. Models with smaller AIC values are more suitable approximations of the data than models with higher AIC values (Akaike, 1998; Burnham \& Anderson, 2004). Thus, we considered the model with the lowest AIC to be the model with the best fit, given the data.

Additionally, we computed Akaike weights for nonredundant models. Akaike weights indicate the relative likelihood of a model in comparison to the other competing models in the model set, given the data (Wagenmakers \& Farrell, 2004) and therefore provide additional information beyond the identification of one 
Table 2

Descriptive Statistics and Zero-Order Correlations among Variables.

\begin{tabular}{|c|c|c|c|c|c|c|c|}
\hline & \multicolumn{2}{|c|}{ Descriptive statistics } & \multicolumn{5}{|c|}{ Correlations } \\
\hline & Mean & SD & 1. & 2. & 3. & 4. & 5 . \\
\hline 1. Chronological age & 64.40 & 9.57 & - & & & & \\
\hline 2. Sex, female ${ }^{a}$ & 0.55 & 0.50 & -.045 & - & & & \\
\hline 3. Subjective age & 55.40 & 12.94 & .621 & -.084 & - & & \\
\hline 4. Life satisfaction & 5.26 & 1.23 & .041 & -.017 & -.087 & - & \\
\hline 5. Subjective age bias ${ }^{\mathrm{b}}$ & -9.00 & 10.26 & -.150 & -.064 & .682 & -.149 & - \\
\hline
\end{tabular}

single best model given the data. The more similar the Akaike weights of several competing models are the higher the model selection uncertainty, that is the data distinguishes between the competing models with lower reliability. To calculate the Akaike weights for non-redundant models, we first checked if the log-likelihood (LL) difference of two nested models was $<1$ (equivalent to an AIC difference of 2 given the same number of parameters; Symonds \& Moussalli, 2011). This criterion indicates an equally good fit for both models. In this case, we excluded the less parsimonious model (i.e. the model with more parameters) from the model set (Humberg, Dufner, et al., 2019).

Lastly, we plotted the response surface of the model with the best fit using its estimated regression coefficients. To aid interpretation, we plotted the LOC and the ridge of the response surface (for simplicity, we did not plot the LOIC for the estimated model, which runs perpendicular to the LOC). As the response surface should only be interpreted in regions where actual data exists, we also projected a bagplot onto the response surface. The bagplot is a bivariate version of the boxplot and consists of two polygons: the inner polygon, which describes the position of the inner $50 \%$ of data points of the bivariate distribution, and the outer polygon, which is formed by inflating the inner polygon by a factor of three.

\section{Results}

Descriptive statistics and zero-order correlations among the observed variables are summarized in Table 2 .

\section{Model comparisons}

First, we examined the full polynomial regression model in order to check for a meaningful association between subjective age, chronological age, and life satisfaction. The full model was significant with $R^{2}=$ $0.041(p=<.001)$, indicating that chronological age, subjective age, and their quadratic and interaction terms explained about $4 \%$ of the variance in life satisfaction.

Second, we fitted all models in the model set to the data. According to the AIC, the increasing optimal margin model with main effects (hypothesis 3.b) fitted the data best (see Table 3). Further, it demonstrated the highest Akaike weight. This indicates that of the models proposed, the increasing optimal margin model with main effects model has the highest probability of fitting the data best, given the data. It was 1.7 times more likely than the optimal margin model with main effects (hypothesis 2.b) and 4.8 times more likely than the optimal margin model-only model (hypothesis 2.a). The models representing the young(er)-is-better perspective (hypothesis 1.a and hypothesis 1.b) and all supplementary models had virtually no support. Inspection of the other fit indices, including the CFI and $R^{2}$, corroborated these results.

Although the increasing optimal margin model with main effects model (hypothesis 3.b) fitted the data best, it is important to note that the weights of this model combined with the optimal margin with main effects model (hypothesis 2.b) and with the optimal marginonly model (hypothesis 2 .a) exceeded 95\%. This indicates with a likelihood of $95 \%$, that the best model of all is one of these three models. Thus, they can each be considered in the range of plausible models. 
Table 3

Model Comparison for the Main Models, Ordered by AIC

\begin{tabular}{|c|c|c|c|c|c|c|c|}
\hline Model & $k$ & AIC & $\Delta \mathrm{AIC}$ & $\begin{array}{l}\text { Akaike } \\
\text { weight }^{\mathrm{a}}\end{array}$ & $\begin{array}{l}\text { Evidence } \\
\text { ratio }^{\mathrm{b}}\end{array}$ & CFI & $R^{2}$ \\
\hline $\begin{array}{l}\text { 3.b) Increasing optimal } \\
\text { margin (with main effect) }\end{array}$ & 4 & 23574.20 & & .55 & & 1.000 & 0.041 \\
\hline $\begin{array}{l}\text { 2.b) Optimal margin (with } \\
\text { main effect) }{ }^{c}\end{array}$ & 3 & 23575.24 & 1.04 & .33 & 1.7 & 0.996 & 0.041 \\
\hline Full $^{\mathrm{d}}$ & 5 & 23576.06 & 1.86 & - & - & 1.000 & 0.041 \\
\hline 2.a) Optimal margin only ${ }^{\mathrm{c}}$ & 2 & 23577.35 & 3.15 & .15 & 4.8 & 0.986 & 0.040 \\
\hline $\begin{array}{l}\text { 3.a) Increasing optimal } \\
\text { margin only }\end{array}$ & 3 & 23578.50 & 4.29 & - & - & 0.985 & 0.040 \\
\hline $\begin{array}{l}\text { 1.b) Young subjective and } \\
\text { older chronological age }\end{array}$ & 2 & 23712.16 & 137.97 & $<.0001$ & $>1000$ & 0.544 & 0.023 \\
\hline $\begin{array}{l}\text { 1.a) Young subjective age } \\
\text { only }\end{array}$ & 1 & 23821.31 & 247.11 & $<.0001$ & $>1000$ & 0.183 & 0.008 \\
\hline Null & 0 & 23876.29 & 302.10 & $<.0001$ & $>1000$ & 0.000 & 0.000 \\
\hline
\end{tabular}

Note. AICs (and Akaike weights if applicable) of the supplementary models, starting with the lowest: $\mathrm{S} 4: \mathrm{AIC}=23805.91(w=<.0001) ; \mathrm{S}^{\mathrm{d}}: \mathrm{AIC}=23823.31 ; \mathrm{S} 2: \mathrm{AIC}=23865.86(w=<.0001) ; \mathrm{S}^{\mathrm{d}}: \mathrm{AIC}$ $=23866.75 ; \mathrm{S}^{\mathrm{d}}: \mathrm{AIC}=23878.29 ; \mathrm{S}^{\mathrm{d}}: \mathrm{AIC}=23878.29$.

${ }^{a}$ Probability that the model is the best model; obtained from model comparisons after excluding the 5 models that were deemed to be redundant (see footnote d).

${ }^{\mathrm{b}}$ Ratio of the Akaike weight in comparison to the best fitting model. Indicates how much more likely the increasing optimal margin model (with main effect) was in comparison to this model.

${ }^{\mathrm{c}}$ Models included in the $95 \%$ confidence set.

${ }^{\mathrm{d}}$ These models were deemed to be redundant and excluded from the model set that was used for the model comparisons using Akaike weights.

$k=$ Number of free parameters in relation to the null model; AIC $=$ Akaike information criterion; $\mathrm{CFI}=$ Comparative fit index; $R^{2}=$ variance in the outcome that is explained by the model.

Sensitivity analyses indicated that our results were robust to different analytic choices, including using the pre-registered exclusion criteria, when deleting two influential observations, and when taking missing data in predictor variables into account using full maximum likelihood estimation (see Supplementary Material, Results $\mathrm{S} 1$ ).

Taken together, these results support that there is a limitation to the subjective age bias. While feeling younger by a certain amount of is associated with the highest levels of life satisfaction, there are downsides of feeling "too young" with respect to life satisfaction. Moreover, there is some evidence that the limitation of feeling younger changes across the adult lifespan. Older adults can maintain a higher discrepancy between their subjective age and their chronological age before experiencing a downturn in life satisfaction compared to middle-aged adults.

\section{Parameter estimates}

Having established that the increasing optimal margin model (with main effects; hypothesis 3.b) is the best-fitting model, we next investigated the parameter estimates in more detail (Table 4). As the parameters of the model are in itself difficult to interpret, we visualized its response surface (Figure 2).

The parameters and their corresponding response surface show three main characteristics. First, the optimal margin effect is reflected in the parameter $\mathrm{C}$, which is significantly different from zero. Accordingly, the response surface clearly shows that the ridge (dotted line) 
a

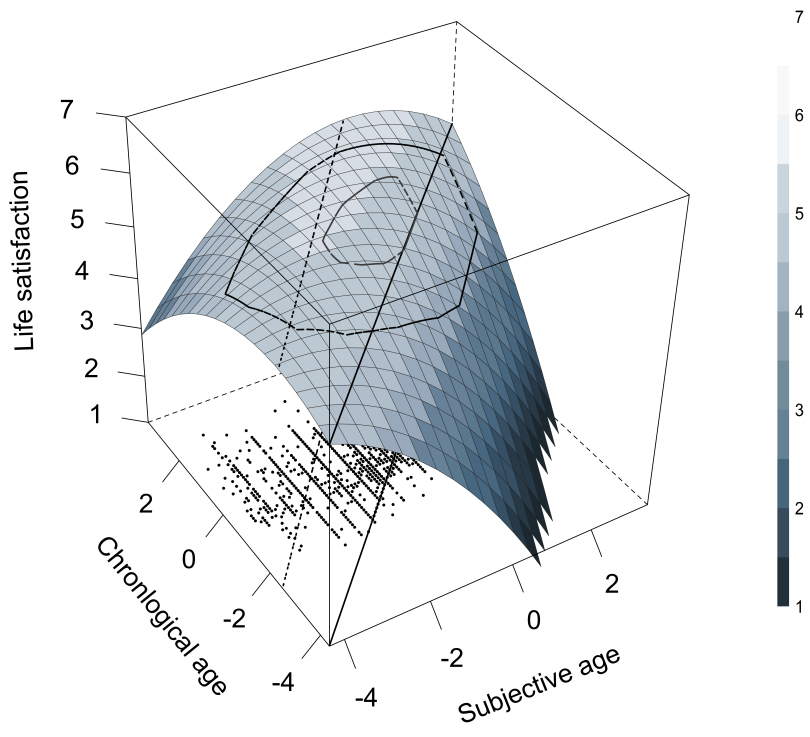

b

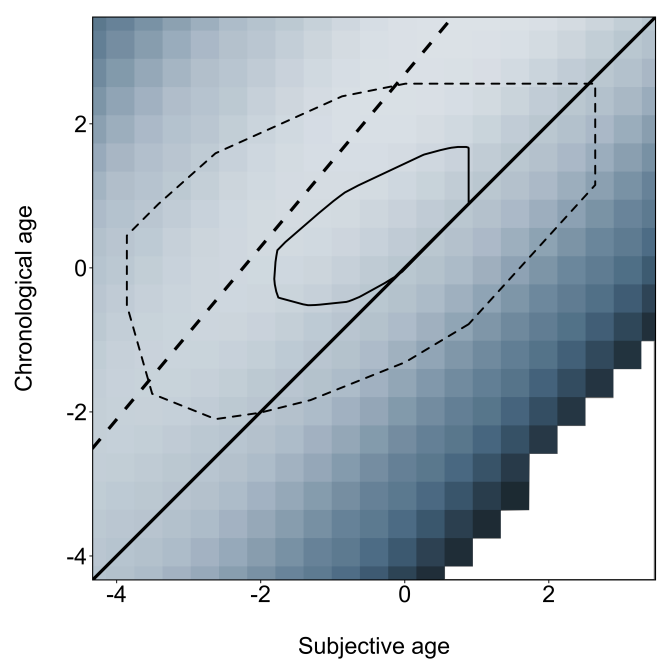

Figure 2. Plots of the response surface of the increasing optimal margin model (with main effects) as estimated based on the data $(N=7,356)$. a. The three-dimensional response surface depicts the associations between subjective age, chronological age, and life satisfaction. Subjective age is on the x-axis (bottom right), chronological age on the y-axis (bottom left), and life satisfaction on the z-axis (vertical). The dots on the floor are projections of the actual data points. b. Contour plot, in which the response surface is projected onto a two-dimensional space. The surfaces are colour-coded so that lighter shades indicate higher levels of life satisfaction and darker shades the lowest levels. The ridge (dotted lines) is shifted away from the line of congruence (LOC; solid line) and slightly rotated, which indicates that there is an optimal margin effect and that the discrepancy at which life satisfaction is maximal increases with increasing chronological age. In addition, a bagplot is projected onto the surfaces, which describes the position of the inner $50 \%$ of points of the bivariate distribution (the inner polygon) and its extension by a factor of three (the outer polygon); the surface should only be interpreted in regions where actual data exists.

is shifted away from the LOC (solid line). These results indicate that life satisfaction is maximized when there is a certain discrepancy between subjective and chronological age. Second, although the parameter $S$, which indicates the rotation of the ridge, was not significantly different from one, model comparisons revealed that it considerably improved the model fit. Graphically, this effect is reflected in a slight rotation of the ridge. These results support the notion that the threshold at which feeling younger is linked to the highest life satisfaction (i.e. the optimal margin) is not fixed but increases throughout adulthood. Finally, the parameter for the mean level effect $b_{\mathrm{M}}$ is significant. Accordingly, the ridge of the response surface is not parallel to the $x-y$ plane, but slightly tilted (only visible in Figure 2a). These results suggest that in addition to the optimal margin effect, the combination of higher subjective age and higher chronological age is linked to higher life satisfaction in comparison to the combination of lower subjective age and lower chronological age.

\section{Quantifying the limit of feeling younger across adulthood}

What specific discrepancy between chronological and subjective age is associated with the highest life satisfaction? To answer this question, we used the parameters of the increasing optimal margin with the main effect model (hypothesis 3.b) to calculate the subjective age bias which the predicted the highest life satisfaction values (see Supplementary Methods for details). Our results revealed that for a person that is 70 years old, for example, a subjective age of 42.8 (i.e. when they feel 27.2 years younger) is associated with the highest levels life satisfaction. For middle-aged people, this discrepancy is slightly lower, while for older people, it is slightly higher (see Table 5). These results, therefore, further illustrate that across adulthood, an increasing subjective age bias is linked to life satisfaction as well as providing a quantification of the point at which one will start to feel "too young". 
Table 4

Regression Coefficients $b_{1}$ to $b_{5}$ and Derived Model Parameters for the Increasing Optimal Margin with Main Effect Model

\begin{tabular}{lrcrrc}
\hline & \multicolumn{1}{c}{ Est. } & $\begin{array}{c}\text { Robust } \\
S E\end{array}$ & $\begin{array}{r}95 \% \mathrm{CI} \\
\text { (lower) }\end{array}$ & $\begin{array}{r}\text { 95\% CI } \\
\text { (upper) }\end{array}$ & $p$ \\
\hline$b_{1}$ & -0.445 & 0.035 & -0.515 & -0.376 & $<.001$ \\
$b_{2}$ & 0.455 & 0.042 & 0.373 & 0.536 & $<.001$ \\
$b_{3}$ & -0.108 & 0.012 & -0.132 & -0.085 & $<.001$ \\
$b_{4}$ & 0.181 & 0.025 & 0.131 & 0.230 & $<.001$ \\
$b_{5}$ & -0.075 & 0.017 & -0.109 & -0.042 & $<.001$ \\
$C$ & 2.737 & 0.358 & -2.036 & -3.439 & $<.001$ \\
$S$ & 1.198 & 0.134 & 0.935 & 1.461 & $.139^{\mathrm{a}}$ \\
$b_{M}$ & 0.083 & 0.031 & 0.023 & 0.143 & .007 \\
\hline
\end{tabular}

Note. The parameters $b_{1}$ to $b_{5}$ have no straightforward interpretation, see main text for more details.

${ }^{\text {a }}$ Tests the null hypothesis that the parameter is $1\left(H_{0}\right.$ : $S=1)$

\section{Discussion}

Our study demonstrates that feeling younger is not always associated with higher levels of life satisfaction, but that there is, throughout adulthood, a limit beyond which feeling younger is detrimental: a discrepancy between one's subjective and one's chronological age is linked to higher life satisfaction when one feely younger up to a certain point, but not more. Consistent with lifespan perspective (Barrett \& Montepare, 2015), this optimal margin of feeling younger changes across adulthood and is greater in older adults. These results extend and refine previous research, which has mostly focused on the young(er)-is-better perspective and suggested a linear relation of subjective age or the discrepancy of subjective and chronological age (e.g. using difference scores) with psychological well-being (Keyes \& Westerhof, 2012; Kleinspehn-Ammerlahn et al., 2008; Stephan et al., 2011; Westerhof \& Barrett, 2005). Instead, our findings reveal a more complex pattern. Feeling younger is only beneficial up to a certain point and feeling younger than one's chronological age

\footnotetext{
${ }^{5}$ An additional analysis was conducted to explore whether the optimal margins differ between men and women. To do so, we computed a multiple-group path model with the specifications of the final model (hypothesis 3.b) and sex as the grouping variable. The results revealed that the model parameters were similar for men and women, except for the main effect of chronological age, which was larger for men than for women. This result is reflected in the differences of the optimal margins across the lifespan. More specifically, the optimal margin increased for both sexes across the lifespan, but this increase was stronger for men than for women (see Supplementary Material; Table S4).
}

to an extreme degree has a negative impact on life satisfaction. In line with previous findings, adults report lower levels of life satisfaction when they feel similar or relative older than their chronological age.

There are several potential reasons why there is a limit beyond which feeling younger becomes detrimental for life satisfaction. While feeling younger may provide benefits due to a dissociation from negative age stereotypes and indicate higher well-being (Stephan et al., 2015; Weiss \& Lang, 2012; Weiss \& Weiss, 2019), extreme distortions from reality might be more detrimental than beneficial. An extremely young subjective age can be considered unrealistic and, thus, might be exhausting and stressful to maintain (Baumeister, 1989; Weiss \& Kornadt, 2018). Furthermore, an exaggerated subjective age bias may reflect that older adults accept negative age-stereotypes against their age-group or unrealistic evaluations of adults' physical and psychological conditions that might become highly problematic and prevent age adaptive processes (Chasteen \& Cary, 2015; Levy et al., 2019; Mock \& Eibach, 2011). For example, previous studies among older adults showed that older subjective ages are linked to more self-regulated driving behavior and plans to stop driving, which can be considered useful from an aging perspective (Barrett \& Gumber, 2019; Pachana et al., 2017). Alternatively, it is conceivable that feeling extremely young may lead to social rejection in the self-perceived age group, which my take a toll on psychological well-being (Chasteen et al., 2011; DeWall \& Bushman, 2011; North \& Fiske, 2013).

Importantly, our findings support a lifespan approach to subjective age (Barrett \& Montepare, 2015). Our results tentatively suggest that the limit up to which feeling younger is beneficial changes across adulthood. Specifically, we found that adults in their 60 s report being most satisfied with their lives when they feel about 25 years younger and adults in their 90s report being most satisfied with their lives when they feel about 30 years younger. It has yet to be determined what accounts for this lifespan pattern of an increasing optimal margin of subjective age.

The current findings open several avenues for future research. First, our study points to the utility of RSA as a method for research on subjective ageing. We have shown, for the first time, that the use of RSA can offer theoretically valuable insights into associations of feeling younger and life satisfaction. As RSA preserves subjective and chronological age as distinct variables and does not collapse them (Edwards, 2002; Humberg, Dufner, et al., 2019), it allows the testing novel and more complex hypotheses on the relations between 
feeling younger and outcomes, as for instance commonly used difference scores allow. Thus, RSA, especially when combined with a model selection approach as in the present study, offers a powerful tool to further elucidate the relations of the subjective age bias with mental and physical health.

A major route for future research is to explore the limits associated with feeling younger in relation to other outcomes. Life satisfaction represents only one aspect of a person's life, albeit arguably an important one. A range of other outcomes, such as depressive symptoms, self-esteem, self-rated health, grip strength, cognitive functioning, or cardiovascular health have previously been linked to subjective age (Alonso Debreczeni \& Bailey, 2020; Keyes \& Westerhof, 2012; Stephan et al., 2018a; Westerhof et al., 2014; Zee \& Weiss, 2019). However, it is unclear whether the findings reported in this study generalize to these other outcomes. While previous studies found that an exaggerated subjective age bias is linked to higher self-esteem, less depressive symptoms, better self-reported health, and fewer functional impairments (Gana et al., 2004; Palgi et al., 2018), they had a number of methodological shortcomings. An important next step would be to re-examine previously reported associations using an RSA framework, so as to map the biopsychosocial domains in which there is a limit to the benefits of feeling younger.

Furthermore, similar studies are now warranted to replicate our findings and extend them to people of other age groups. Although our study provided rich information from mid- to later life, follow-up studies including adolescents, younger adults, and adults over 90 years of age would help to illuminate the relationships between subjective age, chronological age, and life satisfaction across the entire lifespan. For example, up to the age of 25 years, people commonly report a subjective age that is higher than their chronological age (Galambos et al., 1999, 2005; Montepare \& Lachman, 1989; Rubin \& Berntsen, 2006). Thus, future work including younger adults could elucidate whether in this age range, there might be a limit where feeling "too old" becomes detrimental with respect to life satisfaction.

Finally, while our study included younger middleaged adults (younger than 50 years), their sample was relatively small $(\mathrm{N}=205)$. This limitation of our sample may have led to suboptimal estimations in this age range (i.e. it was estimated that among 40-year old adults the greatest life satisfaction was reported when they felt about 18 years old). We would predict that including more younger middle-aged adults might strengthen the evidence for an increasing optimal margin model and provide more reliable predictions for this age range. Future studies should therefore focus on this crucial time of life to replicate our findings and further elucidate the limitations of the subjective age bias in middle adulthood.

Whereas the present study constitutes an important extension of previous findings, three other limitations have to be kept in mind when interpreting our results. First, the cross-sectional data used here does not allow us to make conclusions about the directionality between feeling younger and life satisfaction. Although theories on self-perception propose that self-perceptions are a driver of psychological well-being (Baumeister, 1989), several longitudinal studies suggest that, vice versa, health and well-being may be antecedents of subjective age (Stephan et al., 2018b). Second, subjective age, chronological age, their interaction and quadratic effects explained about $4 \%$ of variance in life satisfaction, which is consistent with previous studies (Spuling et al., 2019; Stephan et al., 2011; Westerhof \& Barrett, 2005). These findings point to the fact that well-being is determined by a large number of factors, and that subjective age represents only one piece of the puzzle. Finally, few individuals $(\mathrm{N}=413$; about $6 \%$ ) fall beyond the optimal margin in the sense that their subjective age falls extremely short of their chronological age. This observation concurs with previous findings demonstrating that few people hold an exaggerated subjective age bias (Gana et al., 2004; Palgi et al., 2018). Our RSA approach, however, shows what can be considered "exaggerated" and feeling how much younger might be "too much" with respect to life satisfaction.

Table 5

Quantifications of the Model-Implied Limit of Feeling Younger Across Different Ages

\begin{tabular}{ccc}
\hline $\begin{array}{c}\text { Chronol. } \\
\text { age }\end{array}$ & $\begin{array}{c}\text { Subj. age with } \\
\text { highest LS }\end{array}$ & $\begin{array}{c}\text { Subj. age bias }^{\mathrm{a}} \\
\text { with highest LS }\end{array}$ \\
\hline 40 & 17.8 & -22.2 \\
50 & 26.1 & -23.9 \\
60 & 34.4 & -25.6 \\
70 & 42.8 & -26.2 \\
80 & 51.1 & -28.9 \\
90 & 59.5 & -30.5 \\
\hline
\end{tabular}

Note. All values in years. Please note that we put optimal in goose feet; LS = life satisfaction ${ }^{\text {a }}$ Calculated from model-implied values as: subjective age at which LS is highest chronological age; negative values indicate feeling younger. 
Beyond refining our understanding of the limits associated with feeling younger in relation to life satisfaction throughout adulthood, our study underscores the importance of formulating theoretical ideas more precisely. Problems of verbal theories in psychology, which are often vague and imprecise, are being identified and pointed out with increasing frequency $(\mathrm{Mu}-$ thukrishna \& Henrich, 2019; Oberauer \& Lewandowsky, 2019). For example, Oberauer and Lewandowsky (2019) argue that often, there is only a weak logical link between theoretical assumptions and the hypotheses that are tested. They suggest that psychological research can be advanced by expressing theoretical assumptions more precisely and even formally, and we believe that our study contributes to this endeavor by formalizing and explicitly comparing hypotheses on the relationship between subjective age, chronological age, and life satisfaction across the adult lifespan.

\section{Conclusion}

In the past decade, numerous studies have suggested that those who feel younger also tend to be happier and healthier, but how exactly is the age we feel, relative to our chronological age, related to our satisfaction across the adult lifespan? Here, we used data from a large sample spanning adulthood and a novel statistical approach (polynomial regression and response surface methodology) to provide clearer insights into this long-standing question. Our results suggest that across the adult lifespan, there is a limitation to feeling younger: those who feel younger up to a certain amount, but no more, report the highest life satisfaction. We also found that the optimal margin of feeling younger increases throughout adulthood, strengthening evidence for a lifespan perspective on subjective ageing.

\section{References}

Akaike, H. (1998). Information theory and an extension of the maximum likelihood principle. In E. Parzen, K. Tanabe, \& G. Kitagawa (Eds.), Selected papers of Hirotugu Akaike (pp. 199-213). Springer. https://doi.org/10.1007/978-1-4612-1694-0_15

Alonso Debreczeni, F., \& Bailey, P. E. (2020). A systematic review and meta-analysis of subjective age and the association with cognition, subjective wellbeing, and depression. The Journals of Gerontology: Series B. https://doi.org/10.1093/geronb/gbaa069

Altman, D. G., \& Royston, P. (2006). The cost of dichotomising continuous variables. BMJ : British Medical Journal, 332(7549), 1080 .
Armenta, B. M., Scheibe, S., Stroebe, K., Postmes, T., \& Van Yperen, N. W. (2018). Dynamic, not stable: Daily variations in subjective age bias and age group identification predict daily well-being in older workers. Psychology and Aging, 33(4), 559571. https://doi.org/10.1037/pag0000263

Barak, B., \& Stern, B. (1986). Subjective age correlates: A research note. The Gerontologist, 26(5), 571-578 https://doi.org/10.1093/geront/26.5.571

Barrett, A. E., \& Gumber, C. (2019). Feeling older and driving less: The effect of age identity on older adults' transition from driving. Innovation in Aging, 3(1). https://doi.org/10.1093/geroni/igz004

Barrett, A. E., \& Montepare, J. M. (2015). "It's about time": Applying life span and life course perspectives to the study of subjective age. In Annual review of gerontology and geriatrics, Vol. 35, 2015: Subjective aging: New developments and future directions (pp. 55-77). Springer Publishing Co.

Baumeister, R. F. (1989). The optimal margin of illusion. Journal of Social and Clinical Psychology, 8(2), 176-189. https://doi.org/10.1521/jscp.1989.8.2.176

Blöchl, M., Nestler, S., \& Weiss, D. (2020, January 22). A limit of the subjective age bias: Feeling younger to a certain degree, but no more, is beneficial for life satisfaction. PsyArXiv. https://doi.org/10.31234/osf.io/pfxqh

Burnham, K. P., \& Anderson, D. R. (2004). Multimodel inference: Understanding AIC and BIC in model selection. Sociological Methods \& Research, 33(2), 261-304. https://doi.org/10.1177/0049124104268644

Burnham, K. P., Anderson, D. R., \& Huyvaert, K. P. (2011). AIC model selection and multimodel inference in behavioral ecology: Some background, observations, and comparisons. Behavioral Ecology and Sociobiology, 65(1), 23-35. https://doi.org/10.1007/s00265-010-1029-6

Chasteen, A. L., Bashir, N. Y., Gallucci, C., \& Visekruna, A. (2011). Age and antiaging technique influence reactions to age concealment. The Journals of Gerontology. Series B, Psychological Sciences and Social Sciences, 66(6), 719-724. https://doi.org/10.1093/geronb/gbr063

Chasteen, A. L., \& Cary, L. A. (2015). Age stereotypes and age stigma: Connections to research on subjective aging. In Annual review of gerontology and geriatrics, Vol. 35, 2015: Subjective aging: New developments and future directions (pp. 99-119). Springer Publishing Co.

Choi, N. G., \& DiNitto, D. M. (2014). Felt age and cognitive-affective depressive symptoms in late life. Aging \& Mental Health, 18(7), 833-837. https://doi.org/10.1080/13607863.2014.886669

Chopik, W. J., \& Giasson, H. L. (2017). Age differences in explicit and implicit age attitudes across the life span. The Gerontologist, 57(suppl_2), S169-S177.https://doi.org/10.1093/geront/gnx058

Cleaver, M., \& Muller, T. E. (2002). I want to pretend I'm eleven years younger: Subjective age and seniors' motives for vacation travel. Social Indicators Research, 60(1/3), 227-241. JSTOR.

DeCoster, J., Iselin, A.-M. R., \& Gallucci, M. (2009). A conceptual and empirical examination of justifications for dichotomization. Psychological Methods, 14(4), 349-366. https://doi.org/10.1037/a0016956

DeWall, C. N., \& Bushman, B. J. (2011). Social acceptance and rejection: The sweet and the bitter. Current Directions in Psychological Science. https://doi.org/10.1177/0963721411417545

Diener, E., Emmons, R. A., Larsen, R. J., \& Griffin, S. (1985). The Satisfaction with Life Scale. Journal of Personality Assessment, 49(1), 71-75. https://doi.org/10.1207/s15327752jpa4901_13 
Edwards, J. R. (2001). Ten difference score myths. Organizational Research Methods, 4(3), 265-287. https://doi.org/10.1177/109442810143005

Edwards, J. R. (2002). Alternatives to difference scores: Polynomial regression analysis and response surface methodology. In Measuring and analyzing behavior in organizations: Advances in measurement and data analysis (pp. 350-400). Jossey-Bass.

Galambos, N. L., Kolaric, G. C., Sears, H. A., \& Maggs, J. L. (1999). Adolescents' subjective age: An indicator of perceived maturity. Journal of Research on Adolescence, 9(3), 309-337. https://doi.org/10.1207/s15327795jra0903_4

Galambos, N. L., Turner, P. K., \& Tilton-Weaver, L. C. (2005). Chronological and subjective age in emerging adulthood: The crossover effect. Journal of Adolescent Research, 20(5), 538556. https://doi.org/10.1177/0743558405274876

Gana, K., Alaphilippe, D., \& Bailly, N. (2004). Positive illusions and mental and physical health in later life. Aging \& Mental Health, $8(1)$

$58-64$. https://doi.org/10.1081/13607860310001613347

Heckhausen, J., Dixon, R. A., \& Baltes, P. B. (1989). Gains and losses in development throughout adulthood as perceived by different adult age groups. Developmental Psychology, 25(1), 109121. https://doi.org/10.1037/0012-1649.25.1.109

Humberg, S., Dufner, M., Schönbrodt, F. D., Geukes, K., Hutteman, R., Küfner, A. C. P., van Zalk, M. H. W., Denissen, J. J. A., Nestler, S., \& Back, M. D. (2019). Is accurate, positive, or inflated self-perception most advantageous for psychological adjustment? A competitive test of key hypotheses. Journal of Personality and Social Psychology, 116(5), 835-859. https://doi.org/10.1037/pspp0000204

Humberg, S., Nestler, S., \& Back, M. D. (2019). Response surface analysis in personality and social psychology: Checklist and clarifications for the case of congruence hypotheses. Social Psychological and Personality Science, 10(3), 409-419. https://doi.org/10.1177/1948550618757600

Keyes, C. L. M., \& Westerhof, G. J. (2012). Chronological and subjective age differences in flourishing mental health and major depressive episode. Aging \& Mental Health, 16(1), 67-74. https://doi.org/10.1080/13607863.2011.596811

Kleinspehn-Ammerlahn, A., Kotter-Gruhn, D., \& Smith, J. (2008). Self-perceptions of aging: Do subjective age and satisfaction with aging change during old age? The Journals of Gerontology Series B: Psychological Sciences and Social Sciences, 63(6), P377-P385. https://doi.org/10.1093/geronb/63.6.P377

Kotter-Grühn, D., Kornadt, A. E., \& Stephan, Y. (2015). Looking beyond chronological age: Current knowledge and future directions in the study of subjective age. Gerontology, 62(1), 86-93. https://doi.org/10.1159/000438671

Lachman, M. E., Röcke, C., Rosnick, C., \& Ryff, C. D. (2008). Realism and illusion in americans' temporal views of their life satisfaction: Age differences in reconstructing the past and anticipating the future. Psychological Science, 19(9), 889-897. https://doi.org/10.1111/j.1467-9280.2008.02173.x

Lang, F. R., Weiss, D., Gerstorf, D., \& Wagner, G. G. (2013). Forecasting life satisfaction across adulthood: Benefits of seeing a dark future? Psychology and Aging, 28(1), 249-261. https://doi.org/10.1037/a0030797

Levy, B. R., Slade, M. D., \& Lampert, R. (2019). Idealization of youthfulness predicts worse recovery among older individuals. Psychology and Aging, 34(2), 202-207. https://doi.org/10.1037/pag0000330
Mock, S. E., \& Eibach, R. P. (2011). Aging attitudes moderate the effect of subjective age on psychological well-being: Evidence from a 10-year longitudinal study. Psychology and Aging, 26(4), 979-986. https://doi.org/10.1037/a0023877

Montepare, J. M., \& Lachman, M. E. (1989). "You're only as old as you feel": Self-perceptions of age, fears of aging, and life satisfaction from adolescence to old age. Psychology and Aging, 4(1), 73-78.

Muthukrishna, M., \& Henrich, J. (2019). A problem in theory. $\mathrm{Na}$ ture Human Behaviour, 3(3), 221-229. https://doi.org/10.1038/s41562-018-0522-1

North, M. S., \& Fiske, S. T. (2013). Act your (old) age: Prescriptive, ageist biases over succession, consumption, and identity. Personality and Social Psychology Bulletin, 39(6), 720-734. https://doi.org/10.1177/0146167213480043

Oberauer, K., \& Lewandowsky, S. (2019). Addressing the theory crisis in psychology. Psychonomic Bulletin \& Review, 26(5), 1596-1618. https://doi.org/10.3758/s13423-019-01645-2

Pachana, N. A., Jetten, J., Gustafsson, L., \& Liddle, J. (2017). To be or not to be (an older driver): Social identity theory and driving cessation in later life. Ageing \& Society, 37(8), 1597-1608. https://doi.org/10.1017/S0144686X16000507

Palgi, Y., Ayalon, L., Avidor, S., Segel-Karpas, D., \& Bodner, E. (2018). On the edge: The association between extreme values of proportional felt-age and functioning. Psychiatry Research, 270, 538-543. https://doi.org/10.1016/j.psychres.2018.10.035

Royston, P., Altman, D. G., \& Sauerbrei, W. (2006). Dichotomizing continuous predictors in multiple regression: A bad idea. Statistics in Medicine, 25(1), 127-141. https://doi.org/10.1002/sim.2331

Rubin, D. C., \& Berntsen, D. (2006). People over forty feel $20 \%$ younger than their age: Subjective age across the lifespan. Psychonomic Bulletin \& Review, 13(5), 776-780. https://doi.org/10.3758/BF03193996

Schoemann, A. M., \& Branscombe, N. R. (2011). Looking young for your age: Perceptions of anti-aging actions. European Journal of Social Psychology, 41(1), 86-95. https://doi.org/10.1002/ejsp.738

Schönbrodt, F. (2016). Testing fit patterns with polynomial regression models. OSF Preprints. https://doi.org/10.31219/osf.io/ndggf

Spuling, S. M., Klusmann, V., Bowen, C. E., Kornadt, A. E., \& Kessler, E.-M. (2019). The uniqueness of subjective ageing: Convergent and discriminant validity. European Journal of Ageing. https://doi.org/10.1007/s10433-019-00529-7

Stephan, Y., Caudroit, J., \& Chalabaev, A. (2011). Subjective health and memory self-efficacy as mediators in the relation between subjective age and life satisfaction among older adults. Aging \& Mental Health, 15(4), 428-436. https://doi.org/10.1080/13607863.2010.536138

Stephan, Y., Sutin, A. R., \& Terracciano, A. (2015). How old do you feel? The role of age discrimination and biological aging in subjective age. PloS One, 10(3), e0119293. https://doi.org/10.1371/journal.pone.0119293

Stephan, Y., Sutin, A. R., \& Terracciano, A. (2018a). Subjective age and mortality in three longitudinal samples. Psychosomatic Medicine, 80(7), 659-664. https://doi.org/10.1097/PSY.0000000000000613

Stephan, Y., Sutin, A. R., \& Terracciano, A. (2018b, November 29). Determinants and implications of subjective age across adulthood and old age. The Oxford Handbook of Integrative Health Science. fordhb/9780190676384.013.7 https://doi.org/10.1093/ox- 
Steptoe, A., Breeze, E., Banks, J., \& Nazroo, J. (2013). Cohort profile: The English Longitudinal Study of Ageing. International Journal of Epidemiology, 42(6), 1640-1648. https://doi.org/10.1093/ije/dys168

Symonds, M. R. E., \& Moussalli, A. (2011). A brief guide to model selection, multimodel inference and model averaging in behavioural ecology using Akaike's information criterion. Behavioral Ecology and Sociobiology, 65(1), 13-21. https://doi.org/10.1007/s00265-010-1037-6

Teuscher, U. (2009). Subjective age bias: A motivational and information processing approach. International Journal of Behavioral Development, 33(1), 22-31. https://doi.org/10.1177/0165025408099487

Wagenmakers, E.-J., \& Farrell, S. (2004). AIC model selection using Akaike weights. Psychonomic Bulletin \& Review, 11(1), 192-196. https://doi.org/10.3758/BF03206482

Ward, R. A. (1977). The impact of subjective age and stigma on older persons. Journal of Gerontology, 32(2), 227-232. https://doi.org/10.1093/geronj/32.2.227

Weiss, D., \& Freund, A. M. (2012). Still young at heart: Negative age-related information motivates distancing from same-aged people. Psychology and Aging, 27(1), 173-180. https://doi.org/10.1037/a0024819

Weiss, D., \& Kornadt, A. E. (2018). Age-stereotype internalization and dissociation: contradictory processes or two sides of the same coin? Current Directions in Psychological Science, 27(6), 477-483. https://doi.org/10.1177/0963721418777743
Weiss, D., \& Lang, F. R. (2012). "They" are old but "I" feel younger: Age-group dissociation as a self-protective strategy in old age. Psychology and Aging, 27(1), 153-163. https://doi.org/10.1037/a0024887

Weiss, D., \& Weiss, M. (2019). Why people feel younger: Motivational and social-cognitive mechanisms of the subjective age bias and its implications for work and organizations. Work, $\mathrm{Ag}$ ing and Retirement, 5(4), 273-280. https://doi.org/10.1093/workar/waz016

Westerhof, G. J., \& Barrett, A. E. (2005). Age identity and subjective well-being: A comparison of the United States and Germany. The Journals of Gerontology. Series B, Psychological Sciences and Social Sciences, 60(3), S129-136. https://doi.org/10.1093/geronb/60.3.s129

Westerhof, G. J., Miche, M., Brothers, A. F., Barrett, A. E., Diehl, M., Montepare, J. M., Wahl, H.-W., \& Wurm, S. (2014). The influence of subjective aging on health and longevity: A metaanalysis of longitudinal data. Psychology and Aging, 29(4), 793802. https://doi.org/10.1037/a0038016

Zee, K. S., \& Weiss, D. (2019). High-quality relationships strengthen the benefits of a younger subjective age across adulthood. Psychology and Aging, 34(3), 374-388. https://doi.org/10.1037/pag0000349 


\section{Supplementary Material for:}

A limit of the subjective age bias: Feeling younger to a certain degree, but no more, is beneficial for life satisfaction

\section{Maria Blöchl*, Steffen Nestler, David Weiss}

To whom correspondence should be addressed. e-mail: mbloechl@uni-muenster.de

This file includes:

Page

Methods S1 Description of Supplementary Models .................. 2

Figure S1 Response Surfaces of the Supplementary Models ........ 3

Figure S2 Plot of Nesting Structure of all Models ................. 4

Tables S1, S2, S3 Results of Sensitivity Analyses .................... $5 \mathrm{ff}$

Methods S2 Quantification of the Optimal Margin ................... 8

Table S4 Sex Differences in the Optimal Margin .................. 9 


\section{Methods S1: Description of Supplementary Models}

The six supplementary models will be described in detail in the following; they are also depicted in Figure S1.

They included three models reflecting effects of chronological age, without any effects of subjective age: One in which higher chronological age has a detrimental (linear) effect, so that people who are older have lower life satisfaction, no matter their subjective age (S1 model); one in which higher chronological age has a beneficial effect, so that people who are older have higher life satisfaction (S2 model); and one in which chronological age has a curvilinear effect, so that people whose chronological age is close to a specific value (e.g. the sample's mean) have the highest life satisfaction (S3 model).

Furthermore, we included two additional variants of the young(er)-is-better perspective: One in which subjective age has a curvilinear effect while chronological age has no effect, so that people whose subjective age is close to a specific value (e.g. the sample's mean) have the highest life satisfaction, regardless of their chronological age (S4 model); and one in which lower chronological age additionally has a beneficial (linear) effect on life satisfaction, so that people who feel younger and are younger have higher life satisfaction (S5 model).

Lastly, we also included a self-knowledge model, which assumes that life satisfaction is highest when people feel exactly their actual age (S6 model). 
Figure S1. Illustrations of Response Surface Graphs for all six Supplementary Hypotheses S1 to S6.

Detrimental chronological age effect

(S1)

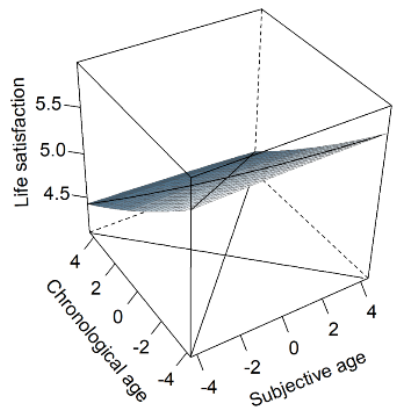

Curvilinear aubjective age effect (S4)

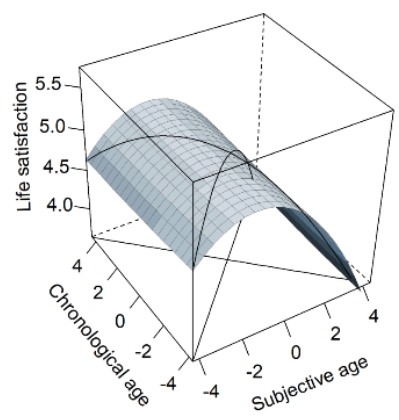

Beneficial chronological age effect

(S2)

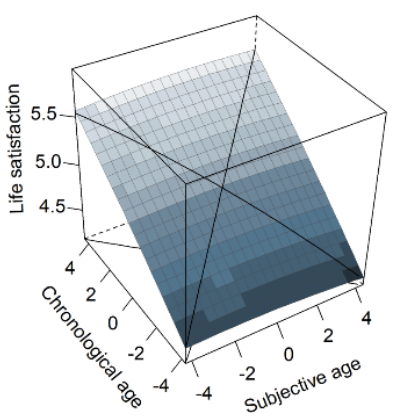

Benefit of feeling younger \& being younger (S5)

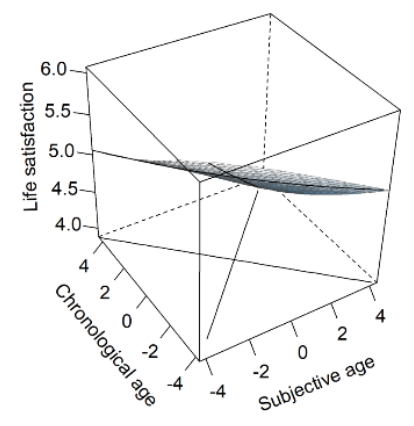

Curvilinear chronological age effect (S3)

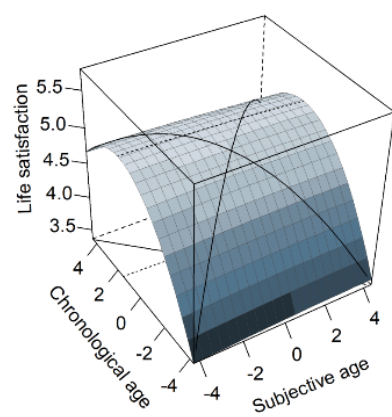

Congrueny / self-knowledge (S6)

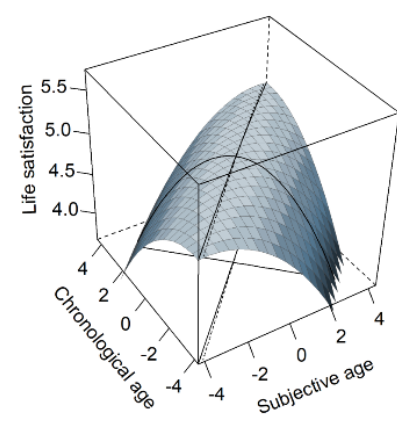




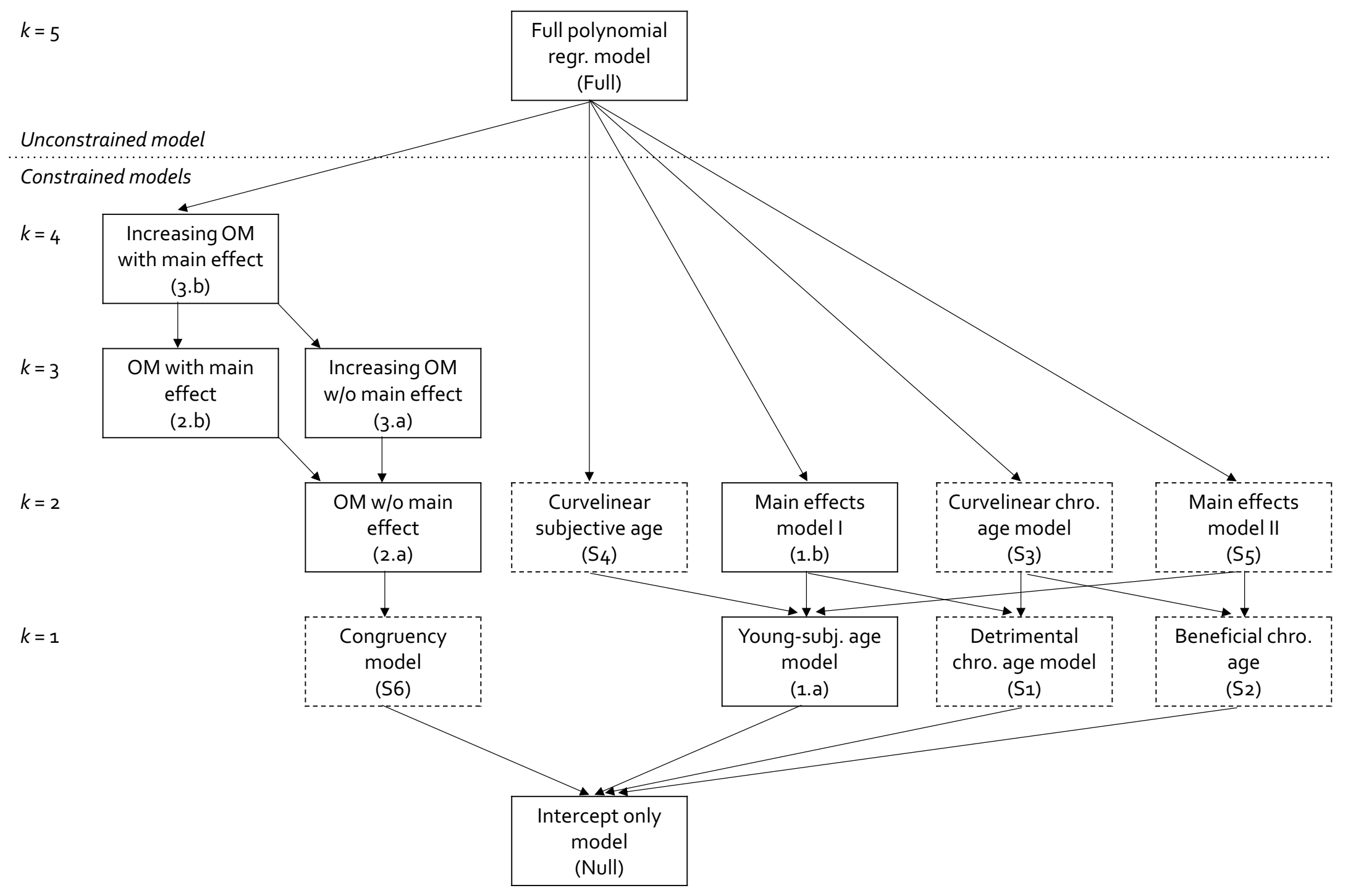

Figure S2. Plot of the nesting of all main (solid frames) and supplementary (dotted frames) models within the full model and each other. $k$ : Number of parameters in comparison to null model; $\mathrm{OM}=$ Optimal margin; w/o $=$ without; chro. $=$ chronological. 


\section{Results S1: Sensitivity Analyses}

To see whether our results were robust to different analytical choices, we conducted several sensitivity analyses.

First, we assessed to what extent our results differed from the pre-registered analyses and our decision to exclude outliers influenced the conclusions. To this end, we ran all models including participants with values of $\pm 3 \mathrm{D}$ of chronological age or subjective age, which were excluded in our main analyses. The results of these analyses did not differ from our main analyses (Table S1).

\section{Table S1}

Results of the Preregistered Analyses Including Participants whose Subjective or Chronological Age were Below or Above 3 SD

\begin{tabular}{|c|c|c|c|c|}
\hline Model & $k$ & AIC & $\Delta \mathrm{AIC}$ & $\begin{array}{l}\text { Akaike } \\
\text { weight }^{\mathrm{a}}\end{array}$ \\
\hline 3.b: Increasing optimal margin (w/me) & 4 & 23855.33 & & .92 \\
\hline Full ${ }^{b}$ & 5 & 23857.11 & 1.77 & - \\
\hline 2.a: Optimal margin-only & 2 & 23860.13 & 4.80 & .08 \\
\hline 2.b: Optimal margin $(\mathrm{w} / \mathrm{me})^{\mathrm{b}}$ & 3 & 23860.45 & 5.12 & - \\
\hline 3.a: Increasing optimal margin-only ${ }^{b}$ & 3 & 23862.13 & 6.80 & - \\
\hline 1.b: Young subj. and high chronol. age & 2 & 23989.71 & 224.84 & $<.0001$ \\
\hline 1.a: Young subj. age-only & 1 & 24080.18 & 277.03 & $<.0001$ \\
\hline Null & 0 & 24149.20 & 293.87 & $<.0001$ \\
\hline
\end{tabular}

Note. $N=7419$. AICs, $\Delta \mathrm{AIC}$ and Akaike weights for the main, the full, and the null models that were non-redundant are provided; all supplementary models were included in these analyses but had virtually no support (results not shown).

a Akaike weights in the sensitivity analyses were calculated as described in the main text (only includes non-redundant models).

${ }^{\mathrm{b}}$ These models were deemed to be redundant and excluded from the model set that was used for model comparisons using Akaike weights. 
Second, we assessed the influence of influential observations on our main analyses. To this end, we examined two global indicators of influence: Cook's $D$ and difference in fits, which both measure the effect of deleting a given observation. Based on evaluation of both indices, we identified two observations that could be considered influential. Deleting those observations did not, however, change the results of our main analyses (Table S2).

\section{Table S2}

Results of the Sensitivity Analyses Excluding Two Influential Observations

\begin{tabular}{lccrr}
\hline \multicolumn{1}{c}{ Model } & $k$ & AIC & $\Delta$ AIC & $\begin{array}{r}\text { Akaike } \\
\text { weight }^{\mathrm{a}}\end{array}$ \\
\hline 3.b: Increasing optimal margin (w/me) & 4 & 23546.37 & & .55 \\
2.b: Optimal margin (w/me) & 3 & 23547.30 & 0.92 & .35 \\
Full & 5 & 23548.23 & 1.85 & - \\
2.a: Optimal margin-only & 2 & 23549.68 & 3.30 & .11 \\
3.a: Increasing optimal margin-only & 3 & 23550.75 & 4.38 & - \\
1.b.: Young subj. and high chronol. age & 2 & 23701.13 & 154.75 & $<.0001$ \\
1.a: Young subj. age-only & 1 & 23813.05 & 266.67 & $<.0001$ \\
Null & 0 & 23869.20 & 322.82 & $<.0001$ \\
\hline
\end{tabular}

Note. $N=7354$. AICs, $\triangle \mathrm{AIC}$ and Akaike weights for the main, the full, and the null models that were non-redundant are provided; all supplementary models were included in these analyses but had virtually no support (results not shown).

a Akaike weights in the sensitivity analyses were calculated as described in the main text (only includes non-redundant models).

${ }^{\mathrm{b}}$ These models were deemed to be redundant and excluded from the model set that was used for model comparisons using Akaike weights. 
Lastly, we re-ran our main analyses with participants with missing data using a full information maximum likelihood estimator (FIML). Under the assumption that data are missing at random, FIML accounts for missing values of the predictor variables and might provide less biased parameter estimates and standard errors. The results using the FIMLprocedure did, however, not differ from the results of our main analyses (Table S3).

\section{Table S3}

Results of the sensitivity analyses using FIML to account for missing data in the predictor variables

\begin{tabular}{lccrr}
\hline Model & $k$ & AIC & $\Delta$ AIC & $\begin{array}{r}\text { Akaike }_{\text {weight }^{\mathrm{a}}} \\
\hline \text { Increasing optimal margin (with main }\end{array}$ \\
effect) & 4 & 24399.93 & & .45 \\
Optimal margin (with main effect) & 3 & 24400.27 & 0.34 & .37 \\
Optimal margin-only & 2 & 24401.79 & 1.86 & .18 \\
Full & 5 & 24401.86 & 1.93 & - \\
Increasing optimal margin-only & 3 & 24402.95 & 3.03 & - \\
Low subjective and high chronological age & 2 & 24526.13 & 126.20 & $<.0001$ \\
Low subjective age-only & 1 & 24630.99 & 231.06 & $<.0001$ \\
Null & 0 & 24685.47 & 285.54 & $<.0001$ \\
\hline
\end{tabular}

Note. $N=7649$. AICs, $\triangle \mathrm{AIC}$ and Akaike weights for the main, the full, and the null models are provided; all supplementary models were included in these analyses but had virtually no support (results not shown).

${ }^{a}$ Akaike weights in the sensitivity analyses were calculated as described in the main text (only includes non-redundant models).

${ }^{\mathrm{b}}$ These models were deemed to be redundant and excluded from the model set that was used for model comparisons using Akaike weights. 


\section{Methods S2: Calculating Optimal Levels of Subjective Age Bias across Adulthood}

In the following, we will detail how we calculated at which subjective ages people's life satisfaction is highest for different levels of chronological age. First, we obtained parameter estimates for the first principle axis, which is the projection of the ridge onto the $\mathrm{x}-\mathrm{y}$ plane (see Humberg, Nestler, \& Back, 2019; Schönbrodt, 2016 for details and formulas). The first principle axis can be expressed as

$$
\text { chronological age }=p_{10}+p_{11} \times \text { subjective age }
$$

For the increasing optimal margin (with mean effects) hypothesis, the parameter $p_{11}$ can be obtained by:

$$
p_{11}:=\left(b_{5}-b_{3}+\sqrt{\left(\left(\left(b_{3}-b_{5}\right)^{2}\right) \times\left(b_{4}{ }^{2}\right)\right)}\right) / b_{4}
$$

The parameter $p_{10}$ can then be obtained by

$$
p_{10}:=Y_{0}-p_{11} \times X_{0}
$$

with

$$
\begin{aligned}
& X_{0}:=\left(b_{2} \times b_{4}-2 \times b_{1} \times b_{5}\right) /\left(4 \times b_{3} \times b_{5}-b_{4}^{2}\right) \\
& Y_{0}:=\left(b_{1} \times b_{4}-2 \times b_{2} \times b_{3}\right) /\left(4 \times b_{3} \times b_{5}-b_{4}^{2}\right)
\end{aligned}
$$

In our case, the first principle axis is therefore ${ }^{1}$

$$
\text { chronological age }=2.688+1.198 \times \text { subjective age }
$$

We lastly calculated the optimal subjective age for chronological ages of 40, 50, 60, 70, 80, and 90 years by using the regression for the first principal axis. To this end, we first standardised all these values to the grand mean and pooled standard deviation (see model fit) and back-transformed all resulting subjective age values accordingly.

\footnotetext{
${ }^{1}$ Please note that we obtained defined and estimated all parameters within the model, using the R package 'lavaan'. Details can be found in the accompanying $\mathrm{R}$ scripts on https://osf.io/kg84e/.
} 


\section{Table S4}

Sex Differences in the Model-implied Limit of Feeling Younger Across Different Ages

\begin{tabular}{cccccc}
\hline & \multicolumn{2}{c}{ Men } & & \multicolumn{2}{c}{ Women } \\
\cline { 2 - 3 } \cline { 5 - 6 } $\begin{array}{c}\text { Chronological } \\
\text { age }\end{array}$ & $\begin{array}{c}\text { SA with } \\
\text { highest LS }\end{array}$ & $\begin{array}{c}\text { SA bias }{ }^{\mathrm{a}} \text { with } \\
\text { highest LS }\end{array}$ & & $\begin{array}{c}\text { SA with } \\
\text { highest LS }\end{array}$ & $\begin{array}{c}\text { SA bias }{ }^{\mathrm{a}} \text { with } \\
\text { highest LS }\end{array}$ \\
\hline 40 & 17.07 & -22.93 & & 16.84 & -23.16 \\
50 & 24.51 & -25.49 & & 25.69 & -24.31 \\
60 & 31.94 & -28.05 & & 34.54 & -25.46 \\
70 & 39.38 & -30.62 & & 43.40 & -26.60 \\
80 & 46.82 & -33.18 & & 52.25 & -27.75 \\
90 & 54.26 & -35.74 & 61.10 & -28.90 \\
\hline
\end{tabular}

Note. All values in years. Please note that we put optimal in goose feet; SA = Subjective age; $\mathrm{LS}=$ life satisfaction

${ }^{\text {a }}$ Calculated as: Subjective age at which LS is highest - chronological age; negative values indicate feeling younger. 